\title{
Luto pela morte de um filho: utilização de um protocolo de terapia cognitivo-comportamental
}

\author{
Bereavement for a child: a cognitive-behavioral treatment protocol
}

\author{
Adriana Cardoso de Oliveira e Silva ${ }^{1}$, Antonio Egidio Nardi ${ }^{2}$ \\ ${ }^{1}$ Pós-doutora em Psiquiatria e Saúde Mental, Instituto de Psiquiatria (IPUB), Universidade Federal do Rio de Janeiro (UFRJ), Rio de Janeiro, RJ. Doutora em \\ Psicologia, Instituto de Psicologia (IP), UFRJ. Pesquisadora, Laboratório de Pânico e Respiração, IPUB, UFRJ. Coordenadora, Laboratório de Tanatologia e Psicometria, \\ Universidade Federal Fluminense, Niterói, RJ. Instituto Nacional de Ciência e Tecnologia Translacional em Medicina. ${ }^{2}$ Livre-docente e professor associado, Faculdade \\ de Medicina, Programa de Pós-Graduação em Psiquiatria e Saúde Mental, IPUB, UFRJ. Coordenador, Laboratório de Pânico e Respiração, IPUB, UFRJ. Instituto \\ Nacional de Ciência e Tecnologia Translacional em Medicina. \\ Suporte financeiro: Conselho Nacional de Desenvolvimento Científico e Tecnológico (CNPq).
}

\section{Resumo}

Objetivo: Apresentar um caso de tratamento de luto, decorrente da perda de um filho, com protocolo cognitivista comportamental.

Descrição do caso: Paciente do sexo feminino, 28 anos, casada, que perdeu seu filho mais velho em um acidente 6 semanas antes do primeiro atendimento. A terapia, composta por 12 sessões, envolveu o trabalho das alterações emocionais e cognitivas, a aprendizagem de novas habilidades, o desenvolvimento de estratégias para lidar com as principais queixas somáticas e o treinamento para manejo dos problemas comportamentais. Os resultados foram avaliados utilizando-se os seguintes instrumentos: Beck Depression Inventory (BDI), Beck Anxiety Inventory (BAI), Beck Hopelessness Scale (BHS), os testes de atenção concentrada (AC) e sustentada (AS) e o Questionário de Saúde Geral de Goldberg (QSG). Comentários: A paciente apresentou redução do quadro de depressão, ansiedade e desesperança. Todos os fatores do QSG apresentaram decréscimo, e houve aumento nas medidas de atenção concentrada e sustentada. O tratamento se mostrou efetivo em relação aos fatores apresentados.

Descritores: Atitude frente à morte, terapia cognitiva, terapia comportamental, tanatologia, morte.

\begin{abstract}
Objective: To describe the case of a patient treated for grief and bereavement for a child using a cognitive-behavioral treatment protocol.

Case description: Female patient, 28 years old, married, who had lost her older son in an accident 6 weeks prior to the first treatment session. The protocol comprised 12 sessions and involved treatment of cognitive and emotional symptoms, the learning of new abilities, development of strategies to deal with the main somatic complaints and training aimed at the handling of behavioral problems. Results were evaluated using the following instruments: Beck Depression Inventory (BDI), Beck Anxiety Inventory (BAI), Beck Hopelessness Scale (BHS), concentrated and sustained attention tests, and Goldberg's General Health Questionnaire (GGHQ).

Comments: The patient presented an improvement of depression, anxiety and hopelessness symptoms. All the factors assessed by the GGHQ decreased, whereas concentrated and sustained attention presented an increase in measurements. The treatment protocol adopted proved to be effective based on the factors assessed.
\end{abstract}

Keywords: Attitude to death, cognitive therapy, behavior therapy, thanatology, death.

\section{Correspondência:}

Adriana Cardoso de Oliveira e Silva, Laboratório de Pânico e Respiração, Instituto de Psiquiatria, Universidade Federal do Rio de Janeiro, Rua Visconde de Pirajá, 407/702, CEP 22410-003, Rio de Janeiro, RJ. E-mail: adrianacardosorj@yahoo.com.br

Não foram declarados conflitos de interesse associados à publicação deste artigo.

Copyright (C) Revista de Psiquiatria do Rio Grande do Sul - APRS

Recebido em 08/12/2009. Aceito em 18/02/2010. 


\section{Introdução}

O luto é uma experiência universal e está associado a maior morbidade e mortalidade ${ }^{1}$. Embora esteja incluído na classificação da $4^{\mathrm{a}}$ edição do Manual Diagnóstico e Estatístico de Transtornos Mentais (DSM-IV) ${ }^{2}$, ainda se encontra catalogado no eixo $\mathrm{V}$, relativo à avaliação global de funcionamento (categoria V62.82). Já na $10^{\mathrm{a}}$ edição da Classificação Internacional de Doenças (CID-10) ${ }^{3}$, consta na categoria Z.

Embora estudos recentes busquem investigar a validade da inclusão do luto nos transtornos mentais no DSM-V e na CID-114, ainda são escassos os estudos voltados para o tema, comparativamente ao que é produzido em relação a outros quadros de saúde mental. Isso fica ainda mais evidente quando o foco é na sistematização do tratamento, buscando evidências de sua efetividade.

Como parte da reação à perda, o sujeito poderá manifestar diversos sinais e sintomas ${ }^{5-7}$, tanto cognitivos (descrença, confusão, sensação de presença, preocupação e até mesmo alucinações), emocionais (tristeza, raiva, culpa, ansiedade, solidão, fadiga, choque, anseio pela presença do ser perdido, alívio e desamparo) e comportamentais (comportamento "aéreo", isolamento social, sonhos com a pessoa morta, evitar ou portar objetos que pertenciam a ela, choro e hiperatividade), quanto físicos (queixas de "aperto no peito", "nó na garganta", "vazio no estômago", sensibilidade aumentada a ruídos, "falta de ar", fraqueza, "boca seca", "falta de energia" e despersonalização). Nesse sentido, especial cuidado deve ser dedicado ao diagnóstico diferencial entre luto e quadros depressivos ${ }^{8-10}$.

É frequente a busca por serviços de emergência médica por pessoas que sofreram perdas recentes ${ }^{1,11}$. Pacientes enlutados apresentam maior necessidade de atendimento médico do que a população geral, maior número de internações e maior vulnerabilidade a problemas psicossomáticos ${ }^{9}$.

Este trabalho tem como objetivo apresentar um estudo de caso em que foi utilizado o enfoque da terapia cognitivocomportamental através de protocolo terapêutico padronizado para atendimento de um caso de luto pela perda de um filho.

\section{Descrição do caso}

Paciente do sexo feminino, com 28 anos de idade, casada há 9 anos. Perdeu o filho mais velho, com 7 anos de idade, 6 semanas antes da primeira sessão, sendo a morte decorrente de acidente. Possui outra filha com 5 anos de idade.

Apresentava bom relacionamento com o cônjuge antes do acidente, mas após o mesmo, relata brigas constantes e distanciamento emocional. Menciona que ele "não sente a morte como ela" e "não consegue entender o que ela está passando".

Refere distanciamento dos amigos e incapacidade de retornar ao trabalho, sem o qual a família não teria como manter-se. Fisicamente, a paciente relata fortes dores de estômago, náuseas, "aperto na garganta", "dores no peito", "dor de cabeça", "sensação de cabeça vazia" e enfraquecimento. Relata também perda de 8 quilos no último mês.

Por insistência do marido, nas últimas 2 semanas que precederam o primeiro atendimento, consultou-se com três diferentes médicos, os quais não encontraram qualquer problema de base orgânica que justificasse o desconforto e as referidas dores; o último dos três médicos realizou o encaminhamento para atendimento psicoterápico.

A paciente relata, ainda, choro frequente intercalado com o que chama de "acessos de raiva" nos quais culpa seu marido, a si mesma e a outras pessoas por "terem permitido que acontecesse". Não apresenta interesse por qualquer atividade que antes lhe agradava e passa cerca de 9 horas por dia no quarto do filho morto observando seus objetos e vendo fotos antigas.

Apresenta, também, como queixa, a incapacidade de concentração em qualquer atividade, o esquecimento das "coisas que tinha para fazer" e pensamentos frequentes de morte, principalmente com a intenção de rever o filho perdido.

Acrescenta que, nesse período em que não está indo trabalhar e tem tido dificuldades para sair de casa, amigos e vizinhos têm ajudado com algumas tarefas, como cuidar da filha mais nova e preparar refeições, apesar de "ela não conseguir comer". A filha mais nova não foi levada ao velório ou ao enterro e nenhum dos pais havia dito nada a ela sobre o assunto.

\section{Avaliação do tratamento}

Para a avaliação do progresso obtido com o tratamento, foram utilizadas as escalas de auto-relato Beck Anxiety Inventory (BAI), Beck Depression Inventory (BDI) e Beck Hopelessness Scale (BHS), que medem, respectivamente, intensidade de ansiedade, depressão e desesperança. A classificação foi realizada com base no manual do instrumento ${ }^{12}$.

Utilizamos, ainda, o Questionário de Saúde Geral de Goldberg $(\mathrm{QSG})^{13}$, que procura identificar a gravidade de distúrbios psiquiátricos menores. O QSG trabalha com cinco fatores: 1) estresse psíquico; 2) desejo de morte; 3) desconfiança no próprio desempenho; 4) distúrbios do sono; e 5) distúrbios psicossomáticos, além de escore relativo à saúde geral.

Como complementação, havendo queixa da participante sobre sua capacidade de atenção e concentração, foram utilizados os testes de Atenção Concentrada (AC) $)^{14}$ e Atenção Sustentada $(\mathrm{AS})^{15}$, para avaliar, respectivamente, sua concentração e sua capacidade de manter a atenção em uma tarefa ao longo do tempo.

Foram realizadas 12 sessões de atendimento clínico, com intervalos de 1 semana entre elas, ao longo de 3 meses. Utilizou-se como enfoque a terapia cognitivo-comportamental, através de protocolo padronizado desenvolvido para casos de enlutamento ${ }^{16}$, onde é valorizado o aprendizado de novas habilidades, tanto cognitivas quanto comportamentais, que possibilitem ao sujeito a readaptação ao seu ciclo de vida, 
considerando a necessidade de uma reformulação de papéis no sistema familiar e na sociedade, de modo geral.

No protocolo utilizado, as técnicas são condizentes com os diferentes momentos do sujeito, ou seja, compatíveis com a fase de enlutamento em que ele se encontra. Desse modo, o protocolo de atendimento pode ser, didaticamente, dividido em três grandes blocos, sendo cada um deles equivalente a quatro sessões terapêuticas, ou 1 mês de acompanhamento, respeitando o curso natural do luto e priorizando as demandas de suas especificidades.

Através da função psicoeducativa, é feito o esclarecimento sobre as fases do luto, assim como sobre as alterações cognitivas, fisiológicas e comportamentais consideradas comuns durante esse período, com o objetivo de reduzir os índices de ansiedade. Étrabalhado o reconhecimento da realidade da perda, e o sujeito é estimulado a compartilhar a experiência e elaborar rituais de despedida. São utilizadas técnicas para o controle da ansiedade e da depressão em momentos agudos. O segundo mês apresenta como foco a resolução de problemas pendentes entre o sujeito enlutado e o ser perdido, a criação de uma rede de apoio social, a reorganização do sistema familiar e a redistribuição de papéis. Finalmente, no último bloco, busca-se propiciar a readaptação do sujeito à vida cotidiana, a organização dos horários de atividades semanais, o investimento em novos objetivos de vida e em novas relações, além da prevenção de recaída.

Os dados foram coletados ao longo dos 3 meses de atendimento, sendo aplicadas semanalmente as escalas BDI e BAI. Os instrumentos BHS, AC, AS e QSG foram aplicados apenas no início e no término do protocolo.

A paciente foi informada quanto aos objetivos, procedimentos e finalidade do tratamento, assim como sobre possíveis utilizações dos dados obtidos, sendo assegurado seu direito à privacidade. Foram seguidos todos os procedimentos éticos vigentes, e o estudo foi aprovado por comitê de ética em pesquisa. A paciente assinou o termo de consentimento livre e esclarecido, concordando, inclusive, com a publicação dos dados em periódicos científicos em meio impresso e eletrônico.

\section{Resultados}

A Tabela 1 apresenta os resultados brutos e a classificação de intensidade decorrentes das aplicações das escalas $\mathrm{BDI}, \mathrm{BAI}$ e BHS, os percentis de $\mathrm{AC}$ e AS, os resultados, também em percentil, dos cinco fatores do QSG e do escore de saúde geral, por semana de tratamento.

Houve redução da ansiedade, que, no início do tratamento, mostrava-se grave, apresentando grande redução quando mensurada no meio do período estipulado para o processo terapêutico (moderada) e sendo classificada como leve ao seu término.

Quanto à depressão, inicialmente classificada como grave, notou-se redução para moderada, primeiramente com 22 pontos (meio do tratamento), e para apenas 12 pontos na mensuração ao final do tratamento, embora esse resultado ainda seja considerado moderado. A desesperança, por sua vez, reduziu de moderada para leve.

A paciente mostrou ainda melhora em seus níveis de concentração do início até o final do tratamento, provavelmente devido à redução da ansiedade e da depressão, que poderiam comprometer esse fator, assim como por conta da redução de pensamentos intrusivos relacionados à perda, que comprometiam essa função.

Todos os fatores do QSG apresentaram redução, sendo marcada a diminuição do percentil referente ao desejo de morte (100 para 45) e do escore de saúde geral, que representa a gravidade da ausência de saúde mental (90 para 45). Fatores orgânicos também mostraram melhoras com o tratamento, com diminuição dos percentis ligados a distúrbios do sono (100 para 55) e a problemas psicossomáticos (95 para 55).

Uma melhora na confiança quanto ao próprio desempenho também foi observada (QSG-3 com queda de 85 para $35)$, o que se revela importante por ajudar a paciente a conseguir elaborar novos projetos, estimulando o novamente o investimento na vida.

Tabela 1 - Resultados para os diferentes instrumentos aplicados e escore de saúde geral

\begin{tabular}{lccc}
\hline & \multicolumn{3}{c}{ Semana de tratamento } \\
\cline { 2 - 3 } Instrumento & $\mathbf{1}$ & $\mathbf{4}$ & $\mathbf{1 2}$ \\
\hline BDI & $46(\mathrm{G})$ & $34(\mathrm{M})$ & $20(\mathrm{M})$ \\
BAI & $42(\mathrm{G})$ & $22(\mathrm{M})$ & $12(\mathrm{~L})$ \\
BHS & $10(\mathrm{M})$ & - & $5(\mathrm{~L})$ \\
AC & 25 & - & 70 \\
AS & 20 & - & 50 \\
QSG-1 & 90 & - & 60 \\
QSG-2 & 100 & - & 45 \\
QSG-3 & 85 & - & 35 \\
QSG-4 & 100 & - & 55 \\
QSG-5 & 95 & - & 55 \\
Saúde geral & 90 & - & 45 \\
\hline
\end{tabular}

$\mathrm{AC}=$ atenção concentrada; $\mathrm{AS}=$ atenção sustentada; $\mathrm{BAI}=$ Beck Anxiety Inventory; $\mathrm{BDI}=$ Beck Depression Inventory; BHS = Beck Hopelessness

Scale; $\mathrm{G}$ = grave; $\mathrm{L}=$ leve; $\mathrm{M}=$ moderado; $\mathrm{QSG}-1$ = estresse psíquico; $\mathrm{QSG}-2$ = desejo de morte; $\mathrm{QSG}-3$ = desconfiança no próprio desempenho;

QSG-4 = distúrbios do sono; QSG-5 = distúrbios psicossomáticos. 


\section{Conclusão}

Considerando a ausência de protocolos terapêuticos padronizados com eficácia comprovada dirigidos especificamente a perdas de filhos, este trabalho apresentou um caso clínico, com enfoque cognitivista-comportamental, que teve resultados avaliados por meio de instrumentos de medidas que apresentam bons parâmetros psicométricos, sendo válidos e fidedignos, além de possuírem normas adequadas à população na qual nossa paciente está inserida, atestando o progresso obtido.

$\mathrm{O}$ tratamento mostrou-se efetivo em relação aos fatores apresentados, além de colaborar para uma melhor qualidade de vida e facilitar a reinserção social da paciente. A evolução do quadro mostrou-se coerente com o tipo de luto e seus determinantes, apontando para a importância da adequação do modelo de atendimento às particularidades desses casos.

Além de ser um primeiro passo para o melhor entendimento dos fatores terapêuticos empregados nesse tipo particular de luto com base em parâmetros objetivos, o estudo apresentado colabora para a ampliação dos limites da terapia cognitivocomportamental, abrindo um novo campo de trabalhos.

\section{Referências}

1. Stroebe M, Schut H, Stroebe W. Health outcomes of bereavement. Lancet. 2007;370:1960-73.

2. American Psychiatric Association. Manual diagnóstico e estatístico de transtornos mentais. $4^{\mathrm{a}}$ ed. Porto Alegre: Artmed; 2002.

3. Organização Mundial da Saúde. Classificação de transtornos mentais e de comportamento da CID-10: descrições clínicas e diretrizes diagnósticas. Porto Alegre: Artes Médicas; 1993.

4. Prigerson HG, Horowitz MJ, Jacobs SC, Parkes CM, Aslan M, Goodkin K, et al. Prolonged grief disorder: psychometric validation of criteria proposed for DSMV and ICD-11. PLoS Med. 2009;6:1-12.

5. Zisook S, Shear K. Grief and bereavement: what psychiatrists need to know. World Psychiatry. 2009;8:67-74.

6. Hensley PL, Clayton PJ. Bereavement: signs, symptoms, and course. Psychiatr Ann. 2008;38:649-54.

7. Parkes CM. Luto: estudos sobre a perda na vida adulta. São Paulo: Summus; 1998.

8. Karam EG, Tabet CC, Alam D, Shamseddeen W, Chatila Y, Mneimneh Z, et al. Bereavement-related and non-bereavement-related depressions: a comparative field study. J Affect Disord. 2009;112:102-10.

9. Clayton PJ. Bereavement and depression. J Clin Psychiatry. 1990;51:34-8.

10. Ghisolfi ES, Broilo LO, Aguiar RW. Luto e transtorno de ajustamento. In: Kapczinski F. Emergências psiquiátricas. Porto Alegre: Artmed; 2001.

11. Worden JW. Terapia de luto: um manual para o profissional de saúde mental. $2^{\mathrm{a}}$ ed. Porto Alegre: Artes Médicas; 1998.

12. Cunha JC. Manual da versão em português das escalas Beck. São Paulo: Casa do Psicólogo; 2001.

13. Goldberg DP. Questionário de saúde geral: manual técnico. São Paulo: Casa do Psicólogo; 1996.

14. Cambraia SV. Teste AC. $3^{\text {a }}$ ed. São Paulo: Vetor; 2003.

15. Sisto FF, Noronha APP, Lamounier DB, Rueda FJM. Teste de atenção dividida e sustentada. São Paulo: Vetor; 2006.

16. Silva ACO. Atendimento clínico para luto no enfoque da terapia cognitivocomportamental. In: Wielenska RC, org. Sobre comportamento e cognição: desafios, soluções e questionamentos. Santo André: ESETec; 2009. 